\title{
Misclassified TyPe 1 AgNS In THE LOCAL UNIVERSE
}

\author{
Jong-Hak Woo ${ }^{1}$, Ji-Gang Kim ${ }^{1,2}$, Daeseong PArK ${ }^{3}$, Hyun-Jin BaE ${ }^{4}$, Jae-Hyuk Kim ${ }^{2}$, Seung-Eon LeE ${ }^{2}$, \\ SAng Chul Kim ${ }^{5}$, AND Hong-Jin KwON ${ }^{2}$ \\ ${ }^{1}$ Astronomy Program, Department of Physics and Astronomy, Seoul National University, Seoul 151-742, Korea \\ woo@astro.snu.ac.kr \\ ${ }^{2}$ R\&E Program, Gyeonggi Science High School for the Gifted, 68-23 Songjuk, Jangan, Suwon 440-800, Korea \\ ${ }^{3}$ Department of Physics and Astronomy, University of California Irvine, CA, USA \\ ${ }^{4}$ Department of Astronomy and Center for Galaxy Evolution Research, Yonsei University, Seoul 120-749, Korea \\ ${ }^{5}$ Korea Astronomy and Space Science Institute, Daejeon 305-348, Korea
}

Received August 25, 2014; accepted September 19, 2014

\begin{abstract}
We search for misclassified type 1 AGNs among type 2 AGNs identified with emission line flux ratios, and investigate the properties of the sample. Using 4113 local type 2 AGNs at $0.02<z<0.05$ selected from Sloan Digital Sky Survey Data Release 7, we detected a broad component of the H $\alpha$ line with a Full-Width at Half-Maximum (FWHM) ranging from 1700 to $19090 \mathrm{~km} \mathrm{~s}^{-1}$ for 142 objects, based on the spectral decomposition and visual inspection. The fraction of the misclassified type 1 AGNs among type 2 AGN sample is $\sim 3.5 \%$, implying that a large number of missing type 1 AGN population may exist. The misclassified type 1 AGNs have relatively low luminosity with a mean broad H $\alpha$ luminosity, $\log \mathrm{L}_{H \alpha}=40.50 \pm 0.35 \mathrm{erg} \mathrm{s}^{-1}$, while black hole mass of the sample is comparable to that of the local black hole population, with a mean black hole mass, $\log \mathrm{M}_{\mathrm{BH}}=6.94 \pm 0.51 \mathrm{M}_{\odot}$. The mean Eddington ratio of the sample is $\log \mathrm{L}_{\mathrm{bol}} / \mathrm{L}_{\mathrm{Edd}}=-2.00 \pm 0.40$, indicating that black hole activity is relatively weak, hence, AGN continuum is too weak to change the host galaxy color. We find that the O III lines show significant velocity offsets, presumably due to outflows in the narrow-line region, while the velocity offset of the narrow component of the $\mathrm{H} \alpha$ line is not prominent, consistent with the ionized gas kinematics of general type 1 AGN population.
\end{abstract}

Key words: galaxies: active — galaxies: nuclei — galaxies: Seyfert: $\mathrm{H} \alpha$ emission line

\section{INTRODUCTION}

Large-area surveys performed in various wavelengths, i.e., X-ray, optical, near-infrared, and mid-infrared, provide a large sample of active galactic nuclei (AGNs), enabling various statistical studies of actively mass accreting supermassive black holes. In particular, by providing the rest-frame optical and UV spectroscopic properties of more than 100000 AGNs over a large redshift range, the Sloan Digital Sky Survey (SDSS) has dramatically changed our understanding of AGN population (e.g., Abazajian et al. 2009), including the local black hole activity (see Heckman \& Best 2014), AGN luminosity and Eddington ratio functions from low- to high-redshift (e.g., Kelly \& Shen 2013), and the connection of black hole activity to star formation (e.g., Netzer 2009).

However, all surveys have their selection functions, hence, it is challenging to avoid selection biases in providing a complete sample of AGNs. In the case of the SDSS, the color-color diagram based on the imaging survey has been used for selecting potential AGN candidates as spectroscopic follow-up targets. Thus, obscured AGNs, i.e., red AGNs (e.g., Glikman et al. 2007, 2013) and X-ray-bright-optically-normal AGNs (e.g., Hornschemeier et al. 2005), can be easily missed from the survey.
In the optical spectroscopic studies, type 1 and type 2 AGNs are often classified based on the presence or absence of broad-emission lines, which are usually defined having a FWHM larger than $1000 \mathrm{~km} \mathrm{~s}^{-1}$, as initially recognized among nearby Seyfert galaxies (e.g., Seyfert 1943). However, if the broad component of the Balmer lines is relatively weak and/or the narrow component is dominant, then it is likely that type $1 \mathrm{AGNs}$ can be misclassified as type 2 AGNs. In addition, if the AGN continuum is relatively weak compared to the stellar continuum, then these objects are likely to be classified as galaxies rather than AGNs in the color-color diagram.

A number of statistical studies have been performed using the SDSS galaxy catalogues, e.g., the MPAJHU value-added catalog, ${ }^{\mathbf{1}}$ and the KIAS value-added galaxy catalog (Choi et al. 2010), which contain the flux-limited local galaxy sample out to $z \sim 0.2$, to investigate the properties of type 2 AGN populations identified through the emission line flux ratios (Baldwin et al. 1981; Kewley et al. 2006). It has been noticed that some type 2 AGNs show a relatively broad component in the $\mathrm{H} \alpha$ line, while a broad component is often missing in the $\mathrm{H} \beta$ line, presumably due to its weak flux compared to the stellar continuum, suggesting that at least some fraction of type 2 AGNs identified through their 
emission line ratios could be genuine type 1 AGNs. A systematic search for these misclassified type 1 AGNs is yet to be performed (see, e.g., Oh et al. 2011; Bae \& Woo 2014). These misclassified AGNs are interesting targets for further study since they are likely to be low luminosity AGNs since their AGN continuum is relatively weak. At the same time, their host galaxy properties can be easily studied while the mass of the central black hole can be estimated from the broad component of $\mathrm{H} \alpha$ using various single-epoch mass estimators (e.g., Woo \& Urry 2002; Park et al. 2012; Bentz et al. 2013).

In this paper, we search for misclassified type 1 AGNs using a large sample of local type 2 AGNs selected from SDSS DR7, by carefully examining the presence of the broad component of the $\mathrm{H} \alpha$ line. Based on the newly found type $1 \mathrm{AGNs}$, we investigate the properties of black hole activity and the kinematics of the ionized gas. Sample selection and the procedure for identifying type 1 AGNs are described in Sections 2 and 3, respectively. In Section 4, we present the properties of the newly found 142 type $1 \mathrm{AGNs}$ and their gas properties. Discussion and conclusions are presented in Section 5. Throughout the paper, we used the cosmological constants of $H_{0}=70 \mathrm{~km} \mathrm{~s}^{-1} \mathrm{Mpc}^{-1}, \Omega_{m}=0.3$, and $\Omega_{\Lambda}=0.7$.

\section{SAMple Selection}

To select type 2 AGNs in the local universe, we utilized the MPA-JHU catalog, which contains 927552 galaxies from the SDSS DR 7 and their derived properties. We selected low-redshift galaxies (i.e., $0.02<z<0.05$ ) and excluded galaxies with low stellar velocity dispersion (i.e., below the SDSS instrumental resolution, $\sigma<$ $\left.70 \mathrm{~km} \mathrm{~s}^{-1}\right)$ in order to utilize the available stellar velocity dispersion measurements in comparing with the gas kinematics. Among these local galaxies, we selected emission-line objects with signal-to-noise ratio $\mathrm{S} / \mathrm{N} \geq 3$ for $\mathrm{H} \alpha, \mathrm{H} \beta, \mathrm{O}$, and $\mathrm{O}$ III, which were used to classify AGNs in the emission line flux ratio diagram (Baldwin et al. 1981). By using the demarcation line for AGNs and star-forming galaxies, 0.73/[log O I/ $\mathrm{H} \alpha$ $+0.59]+1.33<\log \mathrm{O} \mathrm{III} / \mathrm{H} \beta$ or $\log \mathrm{OI} / \mathrm{H} \alpha>-0.59$ (Kewley et al. 2006), we selected 4113 objects as the type 2 AGN sample.

\section{Analysis}

\subsection{Spectral Decomposition}

In studying type $2 \mathrm{AGNs}$, it is of importance to decompose AGN emission lines from the host galaxy stellar continuum in order to properly measure the flux and width of each AGN emission line. For relatively low luminosity AGNs, the line strength of stellar absorption lines is significantly large, hence the precise measurements of AGN emission line flux requires spectral decomposition of the stellar component, for example, using stellar population models or stellar spectral templates (e.g., Park et al. 2012). In addition, for measuring the kinematics of the ionized gas, it is necessary to measure the systemic velocity of the target galaxy

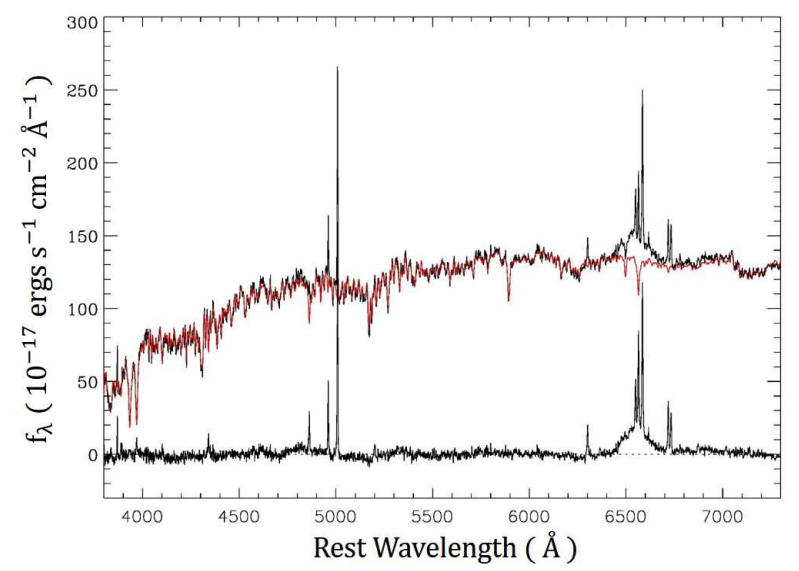

Figure 1. Example of the spectral decomposition for SDSS J140543.16+251352.9. The SDSS spectrum (black line) is modeled with a stellar population model (red line). The residual spectrum at the bottom represents a pure AGN emission line spectrum.

based on the stellar absorption lines. In this study, we used the penalized pixel-fitting (pPXF) method (Cappellari \& Emsellem 2004) to obtain the best fit stellar continuum model, which is based on the 235 simple stellar population models (i.e., 5 different metallicities $\times$ 47 different ages) from the MILES library. First, we de-redshifted all spectra using the redshift value from SDSS. After fitting with the stellar continuum model, we refined the redshift (hence, the systemic velocity) of each object before the emission line fitting procedure. Then, the best-fit continuum model was subtracted from the SDSS spectra, leaving the pure AGN emission-line spectra. This approach is similar to a number of previous studies on the AGN emission and stellar absorption lines (e.g., Woo et al. 2010; Park et al. 2012; Woo et al. 2013). We present an example of the spectral decomposition in Figure 1.

\subsection{Emission Line Fitting}

We examined the SDSS spectra and continuumsubtracted spectra of all 4,113 objects in the type 2 AGN sample, in order to determine whether a broad component is present in the $\mathrm{H} \alpha$ line. In this process, we identified a sample of potential type 1 AGN candidates showing the broad $\mathrm{H} \alpha$ component, based on the visual inspection. Then, we carefully examined the $\mathrm{H} \alpha$ region in the rest-frame $6300-6900 \AA$, by decomposing the broad and narrow components of $\mathrm{H} \alpha$, and $\mathrm{N}$ II lines simultaneously. If the stellar continuum was poorly subtracted from the SDSS spectra due to the low $\mathrm{S} / \mathrm{N}$ ratio, a straight line was adopted to represent the continuum.

Using the MPFIT routine (Markwardt 2009), we modeled each narrow emission line in the $\mathrm{H} \alpha$ region, namely, $\mathrm{H} \alpha \lambda \lambda 6563, \mathrm{~N}$ II $\lambda \lambda 6548,6583$ doublet, and S II $\lambda \lambda 6716,6731$ doublet with a single Gaussian component. In addition, we added a Gaussian component with a FWHM > $1000 \mathrm{~km} \mathrm{~s}^{-1}$, to represent the broad 

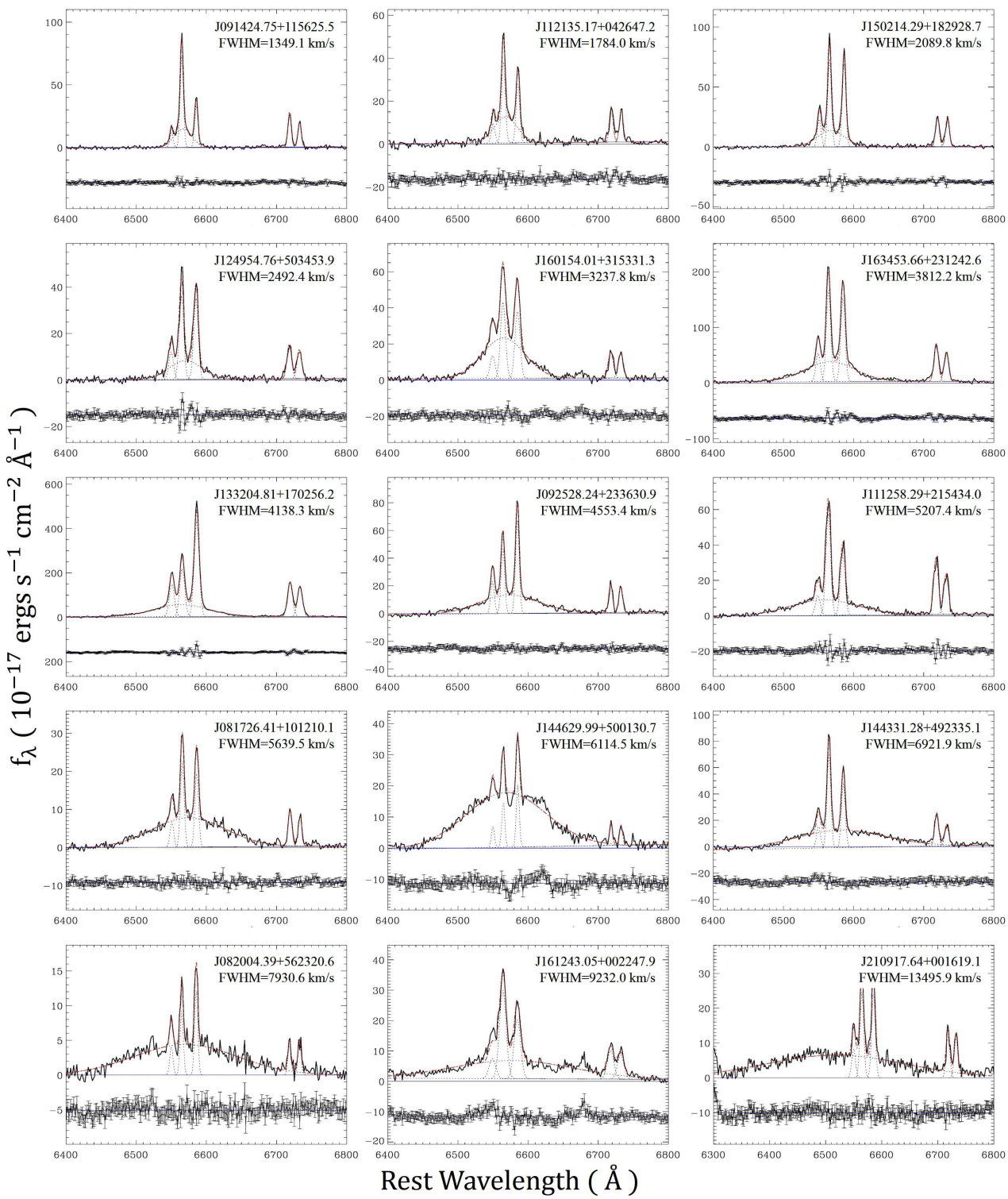

Figure 2. Examples of the best-fit emission line models in the $\mathrm{H} \alpha$ region for 15 objects, are presented in order of increasing FWHM of the broad $\mathrm{H} \alpha$ component. Continuum-subtracted spectra (black lines) are plotted together with the best-fit models (red lines), which are composed of narrow lines ( $\mathrm{N}_{\mathrm{II}}, \mathrm{H} \alpha$, and $\mathrm{S}$ II) and a broad $\mathrm{H} \alpha$ component. At the bottom of each panel the residual is presented (black lines). The object name and the FWHM of the broad H $\alpha$ component are given in each panel.

component of $\mathrm{H} \alpha$. The centers of each Gaussian components for narrow emission lines were fixed relative to each other at their laboratory separations, while the center of the broad $\mathrm{H} \alpha$ component was set to vary in the fitting process. In the case of the line width, we used the same line dispersion value for all narrow emission lines, but for the broad $\mathrm{H} \alpha$ component, we used a free parameter. The line flux ratio of [N II] $\lambda 6583$ and [N II] $\lambda 6548$ is fixed at the theoretical value of 2.96 .

Note that a broad residual from the incorrect subtraction of stellar continuum or the combination of the narrow $\mathrm{H} \alpha$ and $\mathrm{N}$ II line wings could be misinterpreted as a broad-line component. Thus, a careful examination is required to avoid false detection of a broad $\mathrm{H} \alpha$ component. For each object, we carefully examined and compared the raw spectrum and the best-fit model, and conservatively classified the target as type 1 AGNs only if the broad $\mathrm{H} \alpha$ component is clearly needed for the fit. In particular, when the broad $\mathrm{H} \alpha$ is relatively narrow (i.e., FWHM $<\sim 2000 \mathrm{~km} \mathrm{~s}^{-1}$ ), hence we do not see the wing of the broad $\mathrm{H} \alpha$ blueward and redward of $\mathrm{N}$ II in the continuum-subtracted spectra, we removed the target from the list of the type 1 AGN candidates although the best-fit model includes a broad $\mathrm{H} \alpha$ component. Thus, in our conservative classification it is possible that we may miss relatively narrow broad-line objects (e.g., narrow-line Seyfert 1 galaxies). In this process, we identified 142 AGNs, which clearly showed 

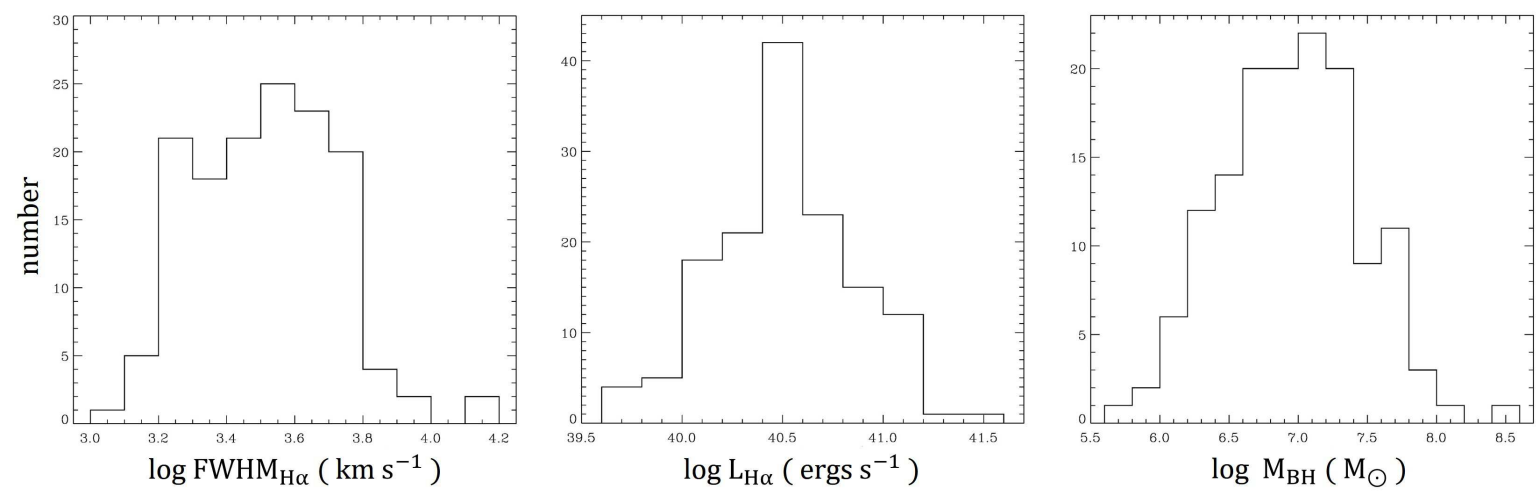

Figure 3. Distribution of the measured $\mathrm{FWHM}_{H \alpha}$ (left), $\log \mathrm{L}_{H \alpha}$ (center), $\log \mathrm{M}_{\mathrm{BH}}$ (right) for 142 misclassified type 1 AGNs.

a broad $\mathrm{H} \alpha$ component. Figure 2 presents examples of the emission line fit in the $\mathrm{H} \alpha$ region for 15 AGNs in increasing magnitude of the FWHM of the broad $\mathrm{H} \alpha$ component.

We measured the line flux and line dispersion of each narrow emission line and the broad $\mathrm{H} \alpha$ line from the best-fit model. We used the luminosity distance using the redshift information in the header of the fits file or the measured redshift from the stellar absorption lines. These two redshifts showed negligible difference in luminosity distance. Figure 3 presents the distributions of the widths and luminosities of the broad $\mathrm{H} \alpha$ component (see Section 4.1 for details).

We also fit the O III line at $5007 \AA$, to measure the velocity center and the velocity dispersion. Since the majority of the AGNs in the sample show a broad wing component in the O III line profile, we used a double Gaussian model to fit the line profile. Then, we measured the flux centroid velocity and velocity dispersion of the best-fit double-Gaussian model.

\subsection{Black Hole Mass and Eddington ratio}

Dynamical black hole mass estimation based on the spatially resolved kinematics is limited to nearby galaxies due to the limited spatial resolution (Kormendy \& Ho 2013). For broad-line AGNs, black hole mass $\left(\mathrm{M}_{\mathrm{BH}}\right)$ can be measured with the reverberation mapping method based on the virial assumption of the gas in the broad-line region (BLR) (Blandford \& McKee 1982). Under the virial relation, black hole mass is expressed as $\mathrm{M}_{\mathrm{BH}}=f V^{2} R_{\mathrm{BLR}} / G$, where $V$ is the characteristic velocity scale of the broad-line gas, typically measured from the line dispersion of the Balmer lines, $f$ is the virial coefficient, which depends on the morphology, orientation, and the kinematics of the BLR, $\mathrm{R}_{\mathrm{BLR}}$ is the size of the BLR measured from the reverberation mapping campaign, and $G$ is the gravitational constant.

Adopting the BLR size - luminosity relation from the recent calibration by Bentz et al. (2013),

$$
R_{B L R}=10^{1.527}\left[\frac{\lambda L_{5100}}{10^{44} \mathrm{erg} \mathrm{s}^{-1}}\right]^{0.533} \text { light days }
$$

the virial relation can be written as follows:

$$
\begin{aligned}
M_{B H}= & f \times 10^{6.817}\left[\frac{V}{10^{3} \mathrm{~km} \mathrm{~s}^{-1}}\right]^{2} \\
& \times\left[\frac{\lambda L_{5100}}{10^{44} \mathrm{erg} \mathrm{s}^{-1}}\right]^{0.533} M_{\odot} .
\end{aligned}
$$

For the misclassified AGNs, we used the line width and luminosity of the broad $\mathrm{H} \alpha$ for estimating black hole masses. Adopting the calibration between the widths of $\mathrm{H} \beta$ and $\mathrm{H} \alpha$ (i.e., $\mathrm{FWHM}_{H \beta}-\mathrm{FWHM}_{H \alpha}$ relation), and the relation between the AGN continuum luminosity and the $\mathrm{H} \alpha$ line luminosity (i.e., $L_{5100}-L_{H \alpha}$ relation) from Greene \& Ho (2005),

$$
\mathrm{FWHM}_{H \beta}=1.07 \times 10^{3}\left[\frac{\mathrm{FWHM}_{H \alpha}}{10^{3} \mathrm{~km} \mathrm{~s}^{-1}}\right]^{1.03} \mathrm{~km} \mathrm{~s}^{-1},
$$

we can derive $\mathrm{M}_{\mathrm{BH}}$ from our measurements based on the spectroscopic decomposition in the $\mathrm{H} \alpha$ region. With the line dispersion $\left(\sigma_{H \alpha}\right)$ and luminosity $\left(\mathrm{L}_{H \alpha}\right)$ of the broad component of $\mathrm{H} \alpha$, the black hole mass can be estimated as follows:

$$
\begin{aligned}
M_{B H}= & f \times 10^{6.565}\left[\frac{\sigma_{H \alpha}}{10^{3} \mathrm{~km} \mathrm{~s}^{-1}}\right]^{2.06} \\
& \times\left[\frac{L_{H \alpha}}{10^{42} \mathrm{erg} \mathrm{s}^{-1}}\right]^{0.46} M_{\odot} .
\end{aligned}
$$

For the virial factor we adopted $f=5.9_{-1.5}^{+2.1}$, which is based on the recent calibration using the combined sample of the quiescent galaxies and reverberation-mapped AGNs (Woo et al. 2013). Note that the systematic uncertainty of the virial factor is $0.31 \mathrm{dex}$ (see Woo et al. 2010) and that black hole mass can easily vary by a factor of 2-3, depending on the virial factor calibration (see Park et al. 2012). Thus, the quoted black hole mass should be treated with caution. In Table 1, we provide the virial product, instead of the black hole mass, which can be determined by multiplying the virial product with the virial factor.

The Eddington luminosity of each object was calculated with the equation $L_{\mathrm{Edd}}=1.25 \times 10^{38} M_{\mathrm{BH}}$ 


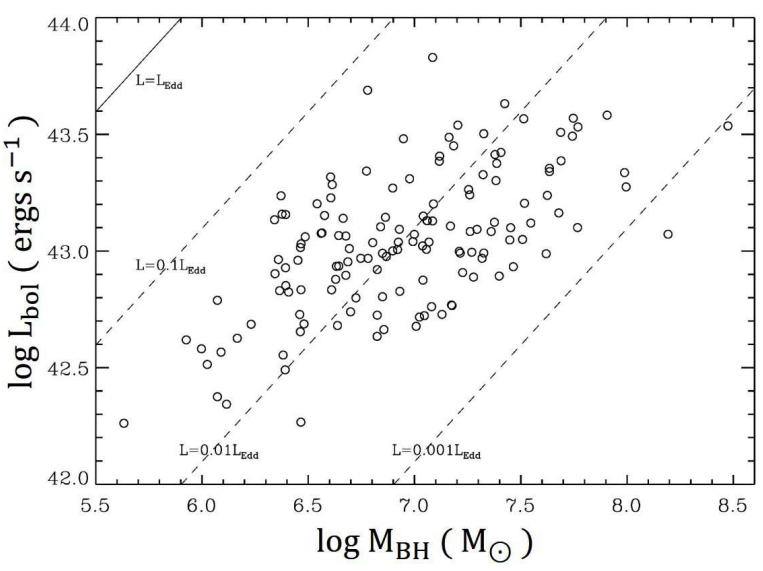

Figure 4. Bolometric luminosity vs. black hole mass for 142 misclassified type 1 AGNs. Dashed lines represent fixed Eddington ratios.

$\left(\mathrm{erg} \mathrm{s}^{-1}\right.$ ) (Wyithe \& Loeb 2002). We used the luminosity of the broad $\mathrm{H} \alpha$ line as a proxy for the AGN bolometric luminosity Utilizing the relation between the luminosity of broad $\mathrm{H} \alpha$ and the continuum luminosity at $5100 \AA$ (Greene \& Ho 2005), and the bolometric correction 9.26 for $\mathrm{L}_{5100}$ (Richards et al. 2006), we derived the bolometric luminosity as follows,

$$
L_{b o l}=2.21 \times 10^{44}\left(\frac{L_{H \alpha}}{10^{42} \mathrm{erg} \mathrm{s}^{-1}}\right)^{0.86} \operatorname{erg~s}^{-1} .
$$

\section{RESULTS}

\subsection{Sample Properties}

Out of the parent sample of 4113 type 2 AGNs in the local universe $(0.02<z<0.05)$, we find a total of 142 misclassified type 1 AGNs based on the presence of the broad component of $\mathrm{H} \alpha\left(\mathrm{FWHM}>1000 \mathrm{~km} \mathrm{~s}^{-1}\right)$. The fraction of misclassified AGNs is $\sim 3.5 \%$ of the parent sample. 25 objects have been previously referenced in the literature (see Greene \& Ho 2004, 2007; Xiao et al. 2013; Reines et al. 2013), indicating a novel discovery of missing type 1 AGNs.

The distribution of the measured properties, i.e., the FWHM and luminosity of the broad $\mathrm{H} \alpha$ component, and black hole mass, are presented in Figure 3. The newly identified type 1 AGNs have a large range of the braod $\mathrm{H} \alpha \mathrm{FWHM}$ velocities, ranging from 1700 to $19090 \mathrm{~km} \mathrm{~s}^{-1}$. The mean line width of broad $\mathrm{H} \alpha$ is $\log \mathrm{FWHM}_{H \alpha}=3.52 \pm 0.21$. In the case of the luminosity, all candidates are relatively low-luminosity AGNs with $\mathrm{H} \alpha$ luminosity lower than $10^{42} \mathrm{erg} \mathrm{s}^{-1}$. The mean broad $\mathrm{H} \alpha$ luminosity is $\log \mathrm{L}_{H \alpha}=40.51 \pm 0.34$. The black hole mass of the sample ranges from $\sim 10^{5.5}$ to $\sim 10^{8.5} \mathrm{M}_{\odot}$ with a mean $\log \mathrm{M}_{\mathrm{BH}} / \mathrm{M}_{\odot}=6.94 \pm 0.51$, while the mean Eddington ratio is $\left.\log \mathrm{L}_{b o l} / \mathrm{L}_{E d d}\right)=$ $-2.00 \pm 0.40$. Compared to the local supermassive black hole population, the misclassified AGNs have a similar black hole mass range with a peak at $\sim 10^{7} \mathrm{M}_{\odot}$ (Heckman \& Best 2014), while the Eddington ratio of the sample is relatively lower than the one of high luminosity QSOs. Table 1 provides the list of 142 misclassified type 1 AGNs along with the measured physical parameters. Figure 4 presents the distribution of the misclassified type 1 AGNs in the $L_{b o l}-M_{B H}$ plane. Overall, the distribution of the sample is similar to that of low luminosity type 1 AGNs (e.g., Woo \& Urry 2002).

\subsection{Comparison with MPA-JHU}

In this section, we compare our new measurements of the $\mathrm{H} \alpha$ line luminosity with that from the the MPAJHU catalog as shown in Figure 5. Since the broad component of $\mathrm{H} \alpha$ was included in our analysis, we expect that our measurements of the narrow $\mathrm{H} \alpha$ line luminosity is reliable, while the MPA-JHU measurements are likely to be overestimated if the broad $\mathrm{H} \alpha$ is not considered in the fitting process.

We find a clear trend that the narrow $\mathrm{H} \alpha$ emission line flux measurements provided by the MPA-JHU catalog is larger than that of our measurement, particularly at the low luminosity regime. The overall overestimation of the narrow $\mathrm{H} \alpha$ line flux can be interpreted as the contribution of the broad $\mathrm{H} \alpha$, since the narrow and broad components were not decomposed, although the line flux can vary depending on how the continuum around the $\mathrm{H} \alpha$ region was determined. The difference is less significant for AGNs with a strong narrow $\mathrm{H} \alpha$ line, presumably due to the weaker contribution of the broad $\mathrm{H} \alpha$ component. These results demonstrate that the line flux measurements of the narrow $\mathrm{H} \alpha$ can be significantly uncertain when a broad component is present and that decomposing and subtracting a broad $\mathrm{H} \alpha$ component from narrow lines (i.e., $\mathrm{H} \alpha$ and $\mathrm{N}$ II) is necessary for misclassified type 1 AGNs.

\subsection{Velocity offset of the emission lines}

We investigate the kinematic properties of the ionized gas, by calculating the velocity offset of the [O III] and the $\mathrm{H} \alpha$ lines with respect to the systematic velocity, which is measured from stellar absorption lines. In the case of $\mathrm{H} \alpha$, we use the narrow and broad components separately, since the physical scale of these two components is clearly different. In Figure 6, we present the distribution of the velocity offset for each line. For the O III line, we used the flux centroid of the line profile as the velocity of the line if a double Gaussian model was used for the fit. The $\mathrm{O}$ III line shows large velocity offsets ranging from $\sim-350$ to $\sim 100 \mathrm{~km} \mathrm{~s}^{-1}$, with a mean $-32 \mathrm{~km} \mathrm{~s}^{-1}$ and $\mathrm{rms} 67 \mathrm{~km} \mathrm{~s}^{-1}$. In addition, the distribution of the $[\mathrm{O} \mathrm{III}]$ velocity offset is asymmetric, indicating that more than a half of the objects has blueshifted [O III] with relatively large velocities. The detected velocity offset with respect to the systemic velocity can be interpreted as due to outflows in the narrow-line region, as various previous studies have used the $\mathrm{O}$ III velocity offset as an outflow indicator (e.g., Boroson 2005; Komossa et al. 2008; Crenshaw et al. 2010; Bae \& Woo 2014).

In the case of the narrow $\mathrm{H} \alpha$ component, most galaxies show relatively small velocity offsets with a mean 

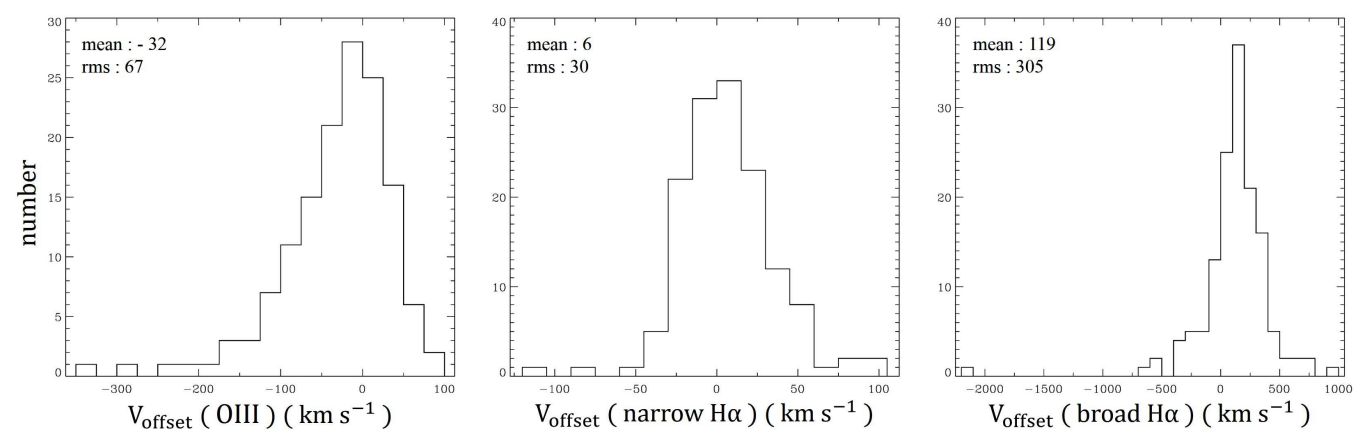

Figure 6. Distribution of the velocity offset with respect to the systemic velocity measured from stellar absorption lines. The $\mathrm{O}$ III line (left) presents on average a larger velocity offset than the narrow component of $\mathrm{H} \alpha$ (center). The broad component of $\mathrm{H} \alpha$ shows a significantly larger velocity offset than the narrow lines (right). In each panel, the mean and rms of the velocity offset is presented.

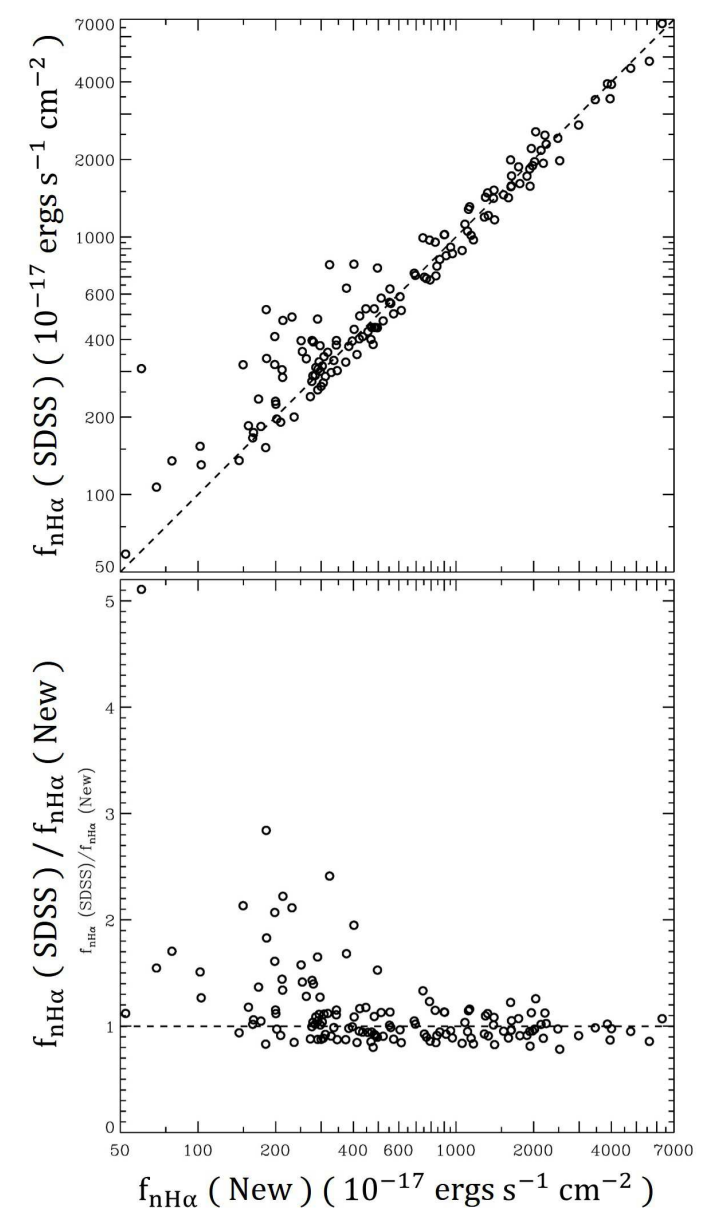

Figure 5. Comparison of the narrow $\mathrm{H} \alpha$ flux measurements of 142 misclassified type 1 AGNs adopted from the MPAJHU catalog and from this study.

$6 \mathrm{~km} \mathrm{~s}^{-1}$ and $\mathrm{rms} 30 \mathrm{~km} \mathrm{~s}^{-1}$. Given the uncertainty of the emission line velocity, which is close to $10-20 \mathrm{~km}$ $\mathrm{s}^{-1}$ as measured from simulated mock spectra based on the SDSS spectra by Bae \& Woo (2014), only a small fraction of the sample seems to show a significant $\mathrm{H} \alpha$ velocity offset. These results of $\mathrm{O}$ III and $\mathrm{H} \alpha$ velocity offsets are consistent with previous studies of type 2 AGNs (Komossa et al. 2008; Bae \& Woo 2014).

In contrast, we detected large velocity offsets in the broad component ofH $\alpha$, with a mean velocity $119 \mathrm{~km}$ $\mathrm{s}^{-1}$ and with a rms of $305 \mathrm{~km} \mathrm{~s}^{-1}$, which is much larger than that of narrow emission lines ( $\mathrm{H} \alpha$ and $\mathrm{O}$ III). The nature of the velocity offset of the broad line is not clear without spatially resolved measurements. We speculate that it may be due to the orbital motion of the black hole and accompanied BLR gas or the inflow/outflow motion of the gas in the BLR. More detailed studies are required to identify the nature of the velocity offset of broad emission lines.

\subsection{Kinematics of the ionized gas}

In Figure 7 we present the velocity dispersion of the ionized gas. For O III we calculated the second moment of the total line profile as the velocity dispersion when we used a double Gaussian model for the fit. In the case of N II and S II, we used the line dispersion of the bestfit Gaussian model. Since we used the same Gaussian model for NiI and SiI, we only present the velocity dispersion of N II.

Compared to the N II lines, the velocity dispersion of O III is much larger, by a factor 1.8 in average. The larger O III velocity dispersion is expected from the presence of a wing component in the line profile. However, once we remove the wing component and measure the velocity dispersion from the narrower core component from the best-fit double Gaussian models (bottom panel in Figure 7), the velocity dispersions of N II and O III become consistent albeit with significant scatter $(\sim 30 \%)$. The mean difference of the velocity dispersion between $\mathrm{N}$ II and the $\mathrm{O}$ III core component is only $\sim 1 \%$, confirming that the core component of $\mathrm{O}$ III and lowionization lines (i.e., N II and S II) have similar kinematics, presumably governed by the gravitational potential of the galaxy bulge (see also Komossa et al. 2008).

In Figure 8 we directly compare the velocity dispersion of N II and the core component of O III with stellar velocity dispersion. The velocity dispersion of N II 

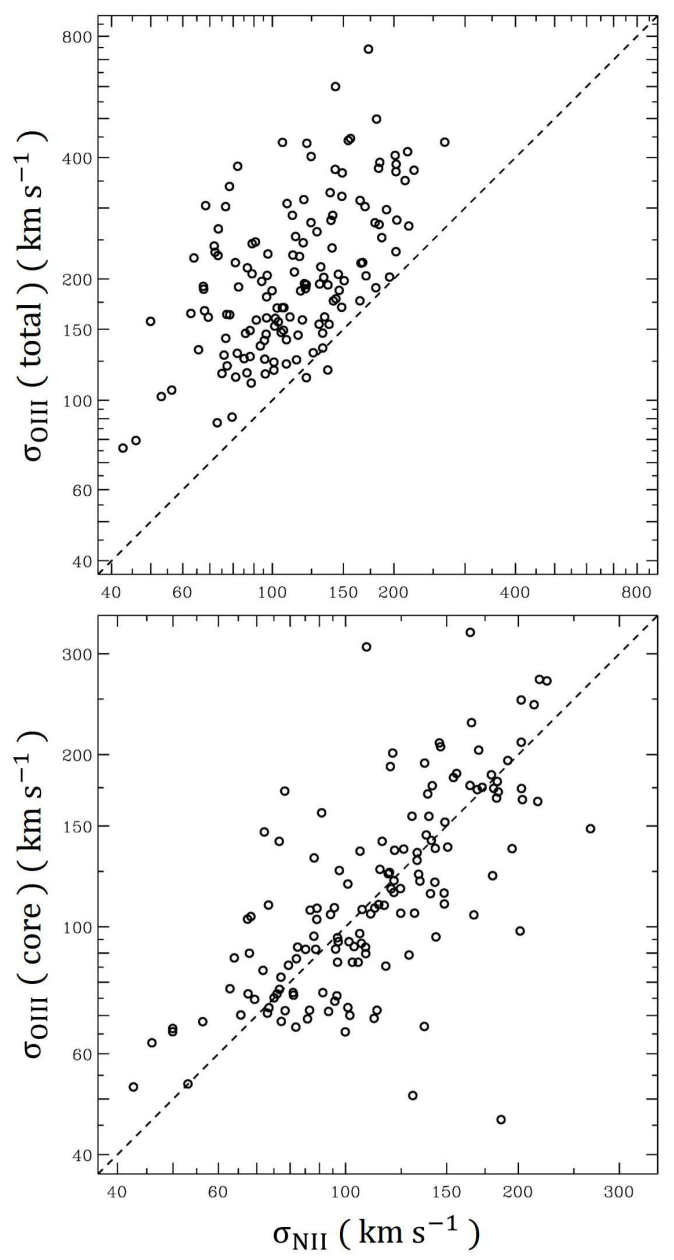

Figure 7. Comparison of the line dispersion of $\mathrm{N}$ II with that of total (top) and core component of [O III] (bottom). While the $\mathrm{O}$ III line is clearly broader than N II due to the strong wing component, the core component of $\mathrm{O}$ III shows consistent line dispersion compared to N II.

is on average smaller than the stellar velocity dispersion by $14 \%$, while the scatter is $\sim 29 \%$. In the case of the core component of $\mathrm{O}$ III (bottom panel in Figure 8), the velocity dispersion is also smaller than the stellar velocity dispersion by $13 \%$, however the scatter is considerably larger $(\sim 43 \%)$. These results suggest that the velocity dispersions of narrow emission lines are on average consistent with stellar velocity dispersions, confirming the results of previous studies (e.g., Nelson \& Whittle 1995). Thus, the width of N II (or O III core component) may be used as a proxy for stellar velocity dispersion. However, if the velocity dispersions of the narrow-emission lines are used instead of the directly measured stellar velocity dispersions, significantly larger uncertainty would be introduced due to the large scatter shown in Figure 8.

The large scatter between emission and absorption lines can be attributed to the contribution from outflow or inflow motion of the gas in the NLR, which seems to preferentially affect the $\mathrm{O}$ III-emitting gas. In addition,
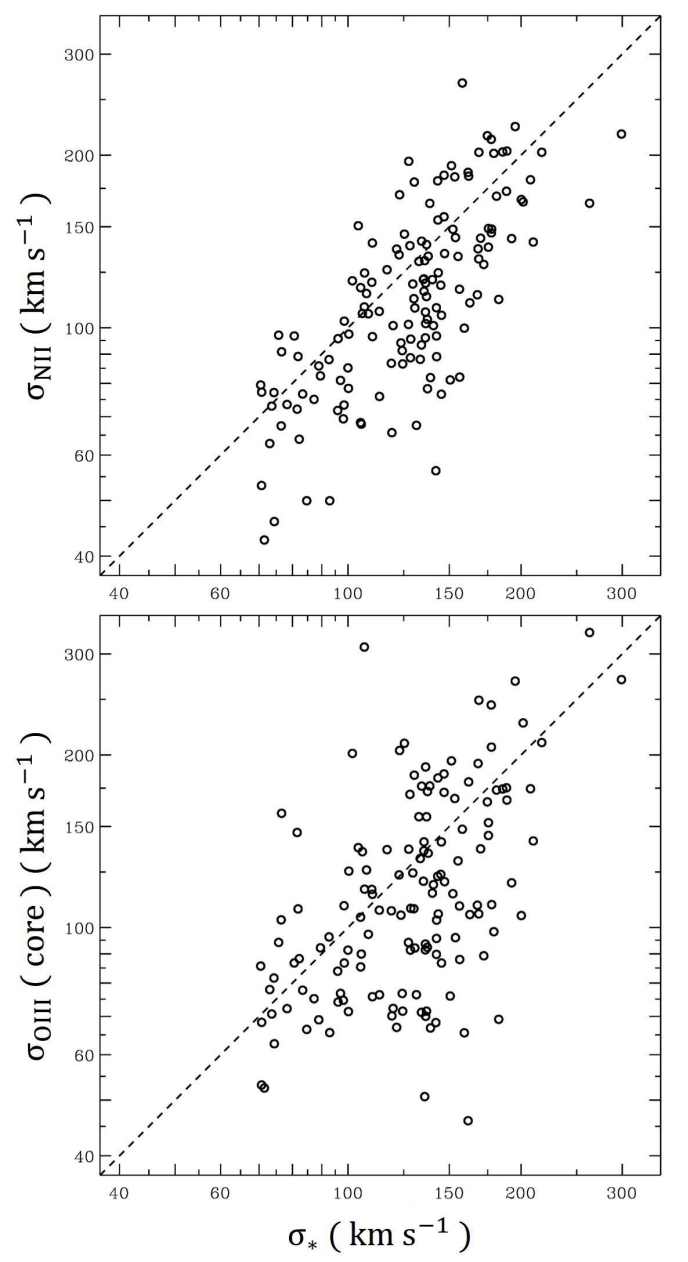

Figure 8. Comparison of the stellar velocity dispersion with the velocity dispersions of $\mathrm{N}$ II (top) and the core component of [O III] (bottom).

we note that the measured stellar velocity dispersion from the SDSS spectra, which were extracted with a $3^{\prime \prime}$ aperture, can suffer from rotational broadening in the stellar absorption lines, depending on the orientation of the stellar disk and the relative strength of the rotation and random velocities as demonstrated by the spatially resolved measurements (Kang et al. 2013; Woo et al. 2013) and the simulated results with various line-of-sight measurement (Bellovary et al. 2014). The larger stellar velocity dispersion relative to the velocity dispersion of N II, by $0.06 \operatorname{dex}(14 \%)$, may indicate an overestimate of the stellar velocity dispersion due to the rotation/inclination effect.

\section{Discussion And Conclusions}

We presented a sample of 142 misclassified type 1 AGNs identified out of a parent sample of 4113 type 2 AGNs at $0.02<z<0.05$, selected from the SDSS DR 7 based on the emission-line flux ratios. The fraction of the misclassified type 1 AGNs among type 2 AGNs is $~ 3.5 \%$; this should be considered as a lower limit because we conservatively identified the broad $\mathrm{H} \alpha$ component by 
Table 1

Physical parameters of 142 missing type 1 AGNs

\begin{tabular}{|c|c|c|c|c|c|c|c|}
\hline $\begin{array}{l}\text { AGN } \\
(1)\end{array}$ & $\begin{array}{c}z \\
(2) \\
\end{array}$ & $\begin{array}{c}\mathrm{FWHM}_{H \alpha} \\
(3) \\
\end{array}$ & $\begin{array}{c}\mathrm{L}_{H \alpha} \\
(4) \\
\end{array}$ & $\begin{array}{c}\mathrm{L}_{b o l} \\
(5)\end{array}$ & $\begin{array}{l}V P \\
(6) \\
\end{array}$ & $\begin{array}{c}\mathrm{L}_{b o l} / L_{E d d} \\
\quad(7)\end{array}$ & $\begin{array}{r}\sigma_{*} \\
(8) \\
\end{array}$ \\
\hline J004730.33+154149.4 & 0.0315 & 3460 & 40.1 & 42.7 & 6.05 & -2.20 & 134 \\
\hline J005847.49-010549.6 & 0.0465 & 5090 & 40.6 & 43.1 & 6.59 & -2.37 & 128 \\
\hline J010606.92+002025.1 & 0.0436 & 2880 & 40.1 & 42.7 & 5.87 & -2.05 & 73 \\
\hline J013402.54-094626.9 & 0.0408 & 4530 & 41.1 & 43.6 & 6.74 & -2.04 & 143 \\
\hline J015612.53+145423.9 & 0.0269 & 1930 & 40.5 & 43.0 & 5.70 & -1.53 & 98 \\
\hline J030834.31+003303.3 & 0.0308 & 3120 & 40.4 & 43.0 & 6.10 & -1.99 & 168 \\
\hline J031142.21+000853.3 & 0.0370 & 12990 & 40.5 & 43.1 & 7.42 & -3.22 & 128 \\
\hline J032525.35-060837.9 & 0.0345 & 2400 & 41.4 & 43.8 & 6.31 & -1.35 & 145 \\
\hline J033957.86-061215.1 & 0.0498 & 4520 & 40.5 & 43.0 & 6.44 & -2.31 & 136 \\
\hline J074507.25+460420.6 & 0.0313 & 6120 & 40.5 & 43.0 & 6.74 & -2.56 & 158 \\
\hline J075151.88+494851.5 & 0.0244 & 2740 & 40.4 & 43.0 & 5.98 & -1.87 & 113 \\
\hline J075217.05+254008.7 & 0.0454 & 2960 & 40.2 & 42.8 & 5.95 & -2.02 & 145 \\
\hline J075643.72+445124.1 & 0.0498 & 3540 & 40.6 & 43.1 & 6.29 & -2.03 & 108 \\
\hline J075828.11+374711.8 & 0.0408 & 2710 & 40.8 & 43.3 & 6.13 & -1.72 & 263 \\
\hline J080421.30+100610.9 & 0.0342 & 3530 & 40.6 & 43.1 & 6.29 & -2.02 & 134 \\
\hline J081726.41+101210.1 & 0.0457 & 5640 & 40.7 & 43.2 & 6.75 & -2.41 & 143 \\
\hline J082004.39+562320.6 & 0.0444 & 7930 & 40.6 & 43.1 & 7.00 & -2.76 & 113 \\
\hline J082351.90+421319.4 & 0.0376 & 1980 & 40.3 & 42.9 & 5.62 & -1.64 & 96 \\
\hline J082414.31+171955.0 & 0.0372 & 2010 & 40.7 & 43.2 & 5.84 & -1.48 & 110 \\
\hline J082620.81+055727.8 & 0.0448 & 3420 & 40.5 & 43.1 & 6.23 & -2.02 & 124 \\
\hline J084137.87+545506.5 & 0.0446 & 3200 & 41.0 & 43.5 & 6.39 & -1.77 & 162 \\
\hline J084143.50+013149.8 & 0.0499 & 3640 & 40.6 & 43.1 & 6.31 & -2.05 & 155 \\
\hline J090554.48+471045.5 & 0.0272 & 2550 & 40.3 & 42.8 & 5.84 & -1.87 & 183 \\
\hline J091330.33+565128.4 & 0.0410 & 2050 & 40.3 & 42.8 & 5.64 & -1.68 & 107 \\
\hline J091424.75+115625.5 & 0.0312 & 1350 & 40.0 & 42.6 & 5.16 & -1.40 & 74 \\
\hline J091708.26+292215.6 & 0.0353 & 3390 & 40.2 & 42.8 & 6.08 & -2.14 & 145 \\
\hline J092313.31+565622.2 & 0.0494 & 2810 & 40.5 & 43.0 & 6.03 & -1.86 & 172 \\
\hline J092528.24+233630.9 & 0.0330 & 4550 & 40.6 & 43.1 & 6.49 & -2.27 & 136 \\
\hline J093106.75+490447.1 & 0.0339 & 1980 & 40.4 & 43.0 & 5.68 & -1.59 & 147 \\
\hline J093551.60+612111.3 & 0.0393 & 2260 & 40.9 & 43.3 & 6.00 & -1.53 & 189 \\
\hline J093917.25+363343.8 & 0.0197 & 4020 & 40.3 & 42.9 & 6.27 & -2.26 & 151 \\
\hline J094319.15+361452.1 & 0.0221 & 2650 & 40.3 & 42.9 & 5.91 & -1.88 & 175 \\
\hline J094830.01+553822.6 & 0.0452 & 2100 & 40.5 & 43.1 & 5.79 & -1.58 & 100 \\
\hline J094931.37+343819.5 & 0.0388 & 2260 & 40.6 & 43.1 & 5.89 & -1.62 & 76 \\
\hline J095009.35+333409.5 & 0.0271 & 5000 & 40.2 & 42.8 & 6.41 & -2.51 & 137 \\
\hline J095437.22+063719.5 & 0.0410 & 6960 & 41.1 & 43.6 & 7.14 & -2.42 & 181 \\
\hline J095742.84+403315.9 & 0.0452 & 1820 & 40.3 & 42.9 & 5.57 & -1.54 & 105 \\
\hline J095824.97+103402.4 & 0.0417 & 4850 & 40.1 & 42.7 & 6.36 & -2.50 & 108 \\
\hline J100207.04+030327.6 & 0.0232 & 4580 & 40.8 & 43.3 & 6.61 & -2.18 & 119 \\
\hline J101439.55-004951.2 & 0.0491 & 3120 & 40.6 & 43.1 & 6.16 & -1.93 & 200 \\
\hline J101640.57+025125.2 & 0.0483 & 4370 & 40.1 & 42.7 & 6.24 & -2.43 & 120 \\
\hline J101833.47+141241.1 & 0.0323 & 3460 & 40.5 & 43.0 & 6.22 & -2.05 & 135 \\
\hline J101846.09+345001.6 & 0.0349 & 1650 & 40.6 & 43.2 & 5.62 & -1.33 & 77 \\
\hline J104243.85+314121.8 & 0.0345 & 3650 & 40.0 & 42.6 & 6.05 & -2.29 & 74 \\
\hline J104250.27+254616.3 & 0.0299 & 3360 & 41.0 & 43.5 & 6.41 & -1.83 & 105 \\
\hline J104546.94+371240.7 & 0.0241 & 3040 & 39.6 & 42.3 & 5.69 & -2.30 & 135 \\
\hline J104809.69+565459.4 & 0.0463 & 3790 & 40.5 & 43.0 & 6.29 & -2.15 & 124 \\
\hline J104930.92+225752.3 & 0.0327 & 2300 & 40.5 & 43.1 & 5.87 & -1.67 & 163 \\
\hline J105214.95+300328.3 & 0.0345 & 1940 & 40.5 & 43.1 & 5.71 & -1.52 & 136 \\
\hline J105427.88+330943.4 & 0.0433 & 1960 & 40.8 & 43.3 & 5.84 & -1.43 & 147 \\
\hline J105756.81+165434.4 & 0.0301 & 6250 & 40.4 & 42.9 & 6.69 & -2.63 & 119 \\
\hline J105833.33+461604.8 & 0.0399 & 3500 & 40.7 & 43.2 & 6.32 & -1.98 & 142 \\
\hline J110501.98+594103.5 & 0.0338 & 2530 & 41.0 & 43.5 & 6.18 & -1.56 & 137 \\
\hline J111106.73+073459.8 & 0.0479 & 4690 & 40.6 & 43.1 & 6.52 & -2.30 & 186 \\
\hline J111117.95+113315.8 & 0.0381 & 5880 & 40.9 & 43.4 & 6.86 & -2.38 & 162 \\
\hline J111258.29+215434.0 & 0.0296 & 5210 & 40.3 & 42.9 & 6.51 & -2.49 & 121 \\
\hline J111349.74+093510.7 & 0.0292 & 3940 & 41.2 & 43.6 & 6.65 & -1.89 & 210 \\
\hline $\mathrm{J} 111653.41+275822.8$ & 0.0347 & 3680 & 40.5 & 43.0 & 6.27 & -2.11 & 104 \\
\hline $\mathrm{J} 111926.22+031205.7$ & 0.0236 & 1960 & 39.7 & 42.3 & 5.34 & -1.87 & 82 \\
\hline J112008.68+341845.8 & 0.0368 & 2540 & 40.3 & 42.9 & 5.86 & -1.85 & 102 \\
\hline
\end{tabular}


Table 1

continued

\begin{tabular}{|c|c|c|c|c|c|c|c|}
\hline $\begin{array}{l}\text { AGN } \\
(1)\end{array}$ & $\begin{array}{c}z \\
(2)\end{array}$ & $\begin{array}{c}\mathrm{FWHM}_{H \alpha} \\
(3)\end{array}$ & $\begin{array}{c}\mathrm{L}_{H \alpha} \\
(4)\end{array}$ & $\begin{array}{l}\mathrm{L}_{b o l} \\
(5)\end{array}$ & $\begin{array}{l}V P \\
(6)\end{array}$ & $\begin{array}{c}\mathrm{L}_{b o l} / L_{E d d} \\
\quad(7)\end{array}$ & $\begin{array}{r}\sigma_{*} \\
(8)\end{array}$ \\
\hline J112011.14+340858.9 & 0.0352 & 2410 & 40.1 & 42.7 & 5.71 & -1.89 & 71 \\
\hline $\mathrm{J} 112135.17+042647.2$ & 0.0470 & 1780 & 40.4 & 43.0 & 5.59 & -1.49 & 100 \\
\hline $\mathrm{J} 112637.73+513423.0$ & 0.0265 & 1760 & 40.0 & 42.6 & 5.39 & -1.64 & 93 \\
\hline $\mathrm{J} 112726.64+264051.5$ & 0.0329 & 4860 & 40.4 & 42.9 & 6.46 & -2.42 & 129 \\
\hline $\mathrm{J} 113355.93+670107.0$ & 0.0397 & 6130 & 40.9 & 43.4 & 6.92 & -2.40 & 156 \\
\hline $\mathrm{J} 113409.01+491516.3$ & 0.0373 & 2410 & 40.1 & 42.7 & 5.69 & -1.91 & 70 \\
\hline $\mathrm{J} 113543.71+490959.8$ & 0.0353 & 2450 & 39.9 & 42.5 & 5.62 & -2.00 & 105 \\
\hline $\mathrm{J} 114223.58+153340.9$ & 0.0444 & 1940 & 40.5 & 43.0 & 5.69 & -1.55 & 82 \\
\hline $\mathrm{J} 114530.25+094344.7$ & 0.0214 & 3080 & 40.4 & 42.9 & 6.05 & -2.00 & 159 \\
\hline $\mathrm{J} 114612.17+202329.9$ & 0.0233 & 5190 & 40.4 & 43.0 & 6.55 & -2.45 & 195 \\
\hline J114840.42-001710.3 & 0.0474 & 3210 & 40.5 & 43.0 & 6.15 & -1.98 & 208 \\
\hline $\mathrm{J} 115246.29+232833.6$ & 0.0220 & 5580 & 40.6 & 43.1 & 6.68 & -2.45 & 169 \\
\hline J115623.23+195932.1 & 0.0409 & 3040 & 40.4 & 43.0 & 6.08 & -1.96 & 136 \\
\hline $\mathrm{J} 120144.91+201941.9$ & 0.0234 & 1940 & 40.3 & 42.8 & 5.59 & -1.63 & 123 \\
\hline J120234.39+544500.7 & 0.0499 & 4050 & 40.6 & 43.1 & 6.40 & -2.16 & 93 \\
\hline $\mathrm{J} 120443.31+311038.2$ & 0.0249 & 4270 & 40.9 & 43.4 & 6.61 & -2.06 & 127 \\
\hline J120704.75+090647.9 & 0.0344 & 6150 & 41.1 & 43.5 & 7.00 & -2.33 & 168 \\
\hline J120908.80+440011.4 & 0.0376 & 2030 & 40.6 & 43.2 & 5.81 & -1.52 & 143 \\
\hline J124054.96+080323.1 & 0.0477 & 1620 & 40.6 & 43.2 & 5.61 & -1.32 & 97 \\
\hline J124240.45+092851.8 & 0.0240 & 2330 & 39.9 & 42.6 & 5.61 & -1.92 & 76 \\
\hline J124610.76+275615.9 & 0.0231 & 4840 & 40.5 & 43.0 & 6.50 & -2.37 & 123 \\
\hline J124954.76+503453.9 & 0.0469 & 2490 & 40.4 & 42.9 & 5.87 & -1.80 & 133 \\
\hline J125258.13+090157.2 & 0.0400 & 3770 & 40.5 & 43.0 & 6.30 & -2.13 & 175 \\
\hline J125552.36+103055.2 & 0.0484 & 4400 & 40.9 & 43.4 & 6.62 & -2.11 & 130 \\
\hline J130340.81+534323.6 & 0.0276 & 1670 & 40.0 & 42.6 & 5.32 & -1.62 & 136 \\
\hline $\mathrm{J} 130342.82+324824.5$ & 0.0367 & 1820 & 40.1 & 42.7 & 5.46 & -1.64 & 74 \\
\hline J130422.19+361543.1 & 0.0443 & 1850 & 41.3 & 43.7 & 6.01 & -1.19 & 137 \\
\hline J130620.97+531823.1 & 0.0237 & 8830 & 40.8 & 43.3 & 7.22 & -2.75 & 141 \\
\hline J130705.01+024337.1 & 0.0477 & 4570 & 40.4 & 43.0 & 6.44 & -2.32 & 147 \\
\hline J130737.68+433117.7 & 0.0353 & 3420 & 40.6 & 43.1 & 6.27 & -1.99 & 83 \\
\hline J132209.96+331005.6 & 0.0373 & 1490 & 40.0 & 42.6 & 5.23 & -1.51 & 78 \\
\hline J132336.84+062424.3 & 0.0394 & 2590 & 40.4 & 43.0 & 5.92 & -1.83 & 85 \\
\hline J133204.81+170256.2 & 0.0215 & 4140 & 40.7 & 43.2 & 6.49 & -2.12 & 152 \\
\hline J134244.42+350346.4 & 0.0243 & 2300 & 40.1 & 42.7 & 5.69 & -1.83 & 87 \\
\hline J134249.94+294546.4 & 0.0431 & 2900 & 40.8 & 43.3 & 6.21 & -1.76 & 170 \\
\hline J135419.95+325547.7 & 0.0261 & 4220 & 40.8 & 43.3 & 6.55 & -2.09 & 177 \\
\hline J135747.63+072346.5 & 0.0236 & 3720 & 40.1 & 42.7 & 6.09 & -2.29 & 100 \\
\hline J140543.16+251352.9 & 0.0298 & 6230 & 40.7 & 43.2 & 6.85 & -2.48 & 139 \\
\hline J140718.28+125313.9 & 0.0274 & 4980 & 40.2 & 42.8 & 6.40 & -2.50 & 139 \\
\hline J140804.00+071939.4 & 0.0239 & 1890 & 40.4 & 42.9 & 5.62 & -1.56 & 82 \\
\hline $\mathrm{J} 141057.23+252950.0$ & 0.0310 & 2400 & 40.5 & 43.1 & 5.91 & -1.71 & 175 \\
\hline J141809.22+073352.3 & 0.0247 & 4500 & 40.2 & 42.8 & 6.31 & -2.42 & 299 \\
\hline J142042.91+262503.2 & 0.0379 & 1900 & 40.8 & 43.3 & 5.83 & -1.38 & 130 \\
\hline J142255.33+325102.3 & 0.0342 & 4060 & 40.8 & 43.3 & 6.48 & -2.09 & 140 \\
\hline J142307.51+283542.3 & 0.0293 & 2810 & 40.6 & 43.1 & 6.07 & -1.83 & 130 \\
\hline J142704.54+355409.5 & 0.0290 & 5930 & 40.9 & 43.3 & 6.86 & -2.39 & 179 \\
\hline J143318.47+344404.4 & 0.0343 & 3180 & 40.5 & 43.0 & 6.13 & -1.99 & 202 \\
\hline $\mathrm{J} 143727.85+254556.0$ & 0.0328 & 1200 & 39.6 & 42.3 & 4.86 & -1.47 & 136 \\
\hline J144331.28+492335.1 & 0.0302 & 6920 & 40.6 & 43.2 & 6.91 & -2.61 & 137 \\
\hline J144629.99+500130.7 & 0.0427 & 6110 & 41.0 & 43.5 & 6.97 & -2.35 & 132 \\
\hline J144837.03+514331.0 & 0.0364 & 2170 & 40.3 & 42.8 & 5.69 & -1.73 & 71 \\
\hline $\mathrm{J} 145048.68+200301.4$ & 0.0437 & 5170 & 40.4 & 43.0 & 6.56 & -2.43 & 154 \\
\hline J150214.29+182928.7 & 0.0477 & 2090 & 40.5 & 43.1 & 5.79 & -1.58 & 81 \\
\hline J150511.42-020831.0 & 0.0372 & 5950 & 40.3 & 42.9 & 6.63 & -2.60 & 138 \\
\hline J150653.38+125131.2 & 0.0216 & 1840 & 39.7 & 42.4 & 5.30 & -1.79 & 106 \\
\hline J150656.41+125048.6 & 0.0224 & 2980 & 40.2 & 42.7 & 5.93 & -2.06 & 217 \\
\hline $\mathrm{J} 151512.25+152412.3$ & 0.0457 & 1540 & 40.7 & 43.2 & 5.60 & -1.23 & 98 \\
\hline J151907.55+260750.6 & 0.0447 & 2520 & 40.5 & 43.0 & 5.92 & -1.78 & 117 \\
\hline $\mathrm{J} 154357.33+283126.4$ & 0.0323 & 1580 & 40.6 & 43.1 & 5.57 & -1.30 & 110 \\
\hline $\mathrm{J} 154744.14+412408.2$ & 0.0326 & 1900 & 40.7 & 43.2 & 5.77 & -1.44 & 89 \\
\hline
\end{tabular}


Table 1

continued

\begin{tabular}{lccccccr}
\hline AGN & $z$ & FWHM $_{H \alpha}$ & $\mathrm{L}_{H \alpha}$ \\
$(1)$ & $(2)$ & $(3)$ & $(4)$ & $\begin{array}{c}\mathrm{L}_{b o l} \\
(5)\end{array}$ & $\begin{array}{c}V P \\
(6)\end{array}$ & $\begin{array}{c}\mathrm{L}_{b o l} / L_{E d d} \\
(7)\end{array}$ & $\begin{array}{r}\sigma_{*} \\
(8)\end{array}$ \\
\hline J155926.11+521235.3 & 0.0423 & 4380 & 40.9 & 43.4 & 6.64 & -2.08 & 134 \\
$\mathrm{~J} 160154.01+315331.3$ & 0.0450 & 3240 & 40.9 & 43.4 & 6.35 & -1.83 & 178 \\
$\mathrm{~J} 160417.30+042135.7$ & 0.0465 & 3660 & 40.3 & 42.8 & 6.16 & -2.20 & 124 \\
$\mathrm{~J} 160445.00+444316.9$ & 0.0433 & 2460 & 40.4 & 42.9 & 5.86 & -1.79 & 107 \\
$\mathrm{~J} 160505.15+452634.8$ & 0.0434 & 5870 & 41.1 & 43.6 & 6.98 & -2.27 & 178 \\
$\mathrm{~J} 160652.16+275539.1$ & 0.0463 & 3250 & 41.1 & 43.5 & 6.43 & -1.76 & 128 \\
$\mathrm{~J} 160746.71+253214.9$ & 0.0406 & 1440 & 40.2 & 42.8 & 5.30 & -1.38 & 96 \\
$\mathrm{~J} 161243.05+002247.9$ & 0.0445 & 9230 & 40.8 & 43.3 & 7.22 & -2.82 & 189 \\
$\mathrm{~J} 161630.67+354228.9$ & 0.0278 & 5730 & 40.5 & 43.0 & 6.68 & -2.50 & 151 \\
$\mathrm{~J} 162131.63+245952.4$ & 0.0378 & 3250 & 40.5 & 43.0 & 6.15 & -2.01 & 110 \\
$\mathrm{~J} 162938.37+384139.2$ & 0.0356 & 1600 & 39.9 & 42.5 & 5.25 & -1.61 & 71 \\
$\mathrm{~J} 163453.66+231242.6$ & 0.0387 & 3810 & 41.0 & 43.5 & 6.55 & -1.92 & 144 \\
$\mathrm{~J} 164107.63+224924.8$ & 0.0339 & 2810 & 40.6 & 43.1 & 6.09 & -1.82 & 89 \\
$\mathrm{~J} 164520.61+424528.0$ & 0.0494 & 5050 & 40.6 & 43.1 & 6.60 & -2.35 & 98 \\
$\mathrm{~J} 171518.57+573931.6$ & 0.0281 & 4340 & 40.1 & 42.7 & 6.25 & -2.40 & 131 \\
$\mathrm{~J} 173159.21+595817.9$ & 0.0292 & 4440 & 40.1 & 42.7 & 6.28 & -2.42 & 153 \\
$\mathrm{~J} 173809.30+584253.6$ & 0.0288 & 6130 & 40.6 & 43.1 & 6.78 & -2.52 & 125 \\
$\mathrm{~J} 204745.25-052515.6$ & 0.0458 & 3200 & 40.9 & 43.4 & 6.35 & -1.81 & 156 \\
$\mathrm{~J} 210917.64+001619.1$ & 0.0500 & 13500 & 41.1 & 43.5 & 7.70 & -3.04 & 169 \\
$\mathrm{~J} 230920.26+004523.3$ & 0.0323 & 5690 & 41.0 & 43.5 & 6.92 & -2.28 & 142 \\
$\mathrm{~J} 232337.44+133908.1$ & 0.0425 & 2850 & 40.4 & 43.0 & 6.01 & -1.91 & 193 \\
$\mathrm{~J} 232611.29+140148.1$ & 0.0462 & 7190 & 40.4 & 43.0 & 6.85 & -2.73 & 143 \\
\hline
\end{tabular}

Notes. Column 1: Object name. Column 2: Redshift. Column 3: FWHM velocity of the broad component of the H $\alpha$ emission line in $\mathrm{km} \mathrm{s}^{-1}$. Column 4: Luminosity of the broad component of the $\mathrm{H} \alpha$ emission line in log scale (erg s${ }^{-1}$ ). Column 5: Bolometric luminosity in $\log$ scale $\left(\mathrm{erg} \mathrm{s}^{-1}\right)$. Column 6: Virial Product in $\log$ scale $\left(\mathrm{M}_{\odot}\right)$. Column 7: Eddington ratio in log scale. Column 8: Stellar velocity dispersion in $\mathrm{km} \mathrm{s}^{-1}$ adopted from the SDSS catalogue.

excluding AGNs that require a broad component with relatively small width. If we extrapolate the fraction of the misclassified type $1 \mathrm{AGNs}, 3.5 \%$, to higher redshift, it is expected that a large number of AGNs could be misclassified as type 2 AGNs in the SDSS DR7 catalog. The discovery of the missing type 1 AGN population will shed light on understanding the low-mass, lowluminosity AGNs and studying AGN population and their properties.

The newly identified type 1 AGN sample has a mean black hole mass $\log \left(\mathrm{M}_{B H} / \mathrm{M}_{\odot}\right)=6.94 \pm 0.51$ based on the most recent single-epoch black hole mass calibrator - although the precise black hole mass values depend on the virial factor. The mean broad $\mathrm{H} \alpha$ luminosity of the sample is $\log \left(\mathrm{L}_{H \alpha} /\left(\mathrm{erg} \mathrm{s}^{-1}\right)=40.50 \pm 0.35\right.$, and the mean Eddington ratio is $\log \mathrm{L}_{b o l} / L_{E d d}=-2.00 \pm 0.40$. The low Eddington ratios of the sample imply that the AGN continuum is too weak to change the host galaxy color, which is consistent with the relatively red color of the targets shown in the SDSS images.

We investigated the velocity offset of each emission line with respect to the systemic velocity measured from stellar absorption lines. We find that the O III lines show relatively large velocity offsets while the velocity offsets of the $\mathrm{H} \alpha$ lines are weaker than for O III, indicating that the $\mathrm{O}$ III-emitting gas is influenced by outflows more strongly. The velocity dispersions of the $\mathrm{N}$ II lines and the $\mathrm{O}$ III core components are consistent with each other and with stellar velocity dispersions, albeit with significant scatter, suggesting that the kine- matics of the gas in the NLR is mainly governed by the large scale gravitational potential (i.e., the galaxy bulge), while outflow/inflow motions can vary the velocity dispersion of narrow emission lines. The kinematic properties of the ionized gas of the sample are similar to those of the general type 1 AGN population, supporting the idea that these objects are simply misclassified type 1 AGNs.

\section{ACKNOWLEDGMENTS}

This work was supported by the National Research Foundation of Korea (NRF) grant funded by the Korean government (MEST; No. 2012R1A2A2A01006087). J. H. W. acknowledges the support by the Korea Astronomy and Space Science Institute (KASI) grant funded by the Korea government (MEST). by the Korea government (No. 2012-006087). J. G. K. acknowledges the support by the 2012 R\&E Program of Gyeonggi Science High School for the Gifted (GSHS).

\section{REFERENCES}

Abazajian, K. N., et al. 2009, The Seventh Data Release of the Sloan Digital Sky Survey, ApJS, 182, 543

Bae, H.-J., \& Woo, J.-H. 2014, A Census of Gas Outflows in Type 2 Active Galactic Nuclei, ApJ, 795, 30

Baldwin, J. A., Phillips, M. M., \& Terlevich, R. 1981, Classification Parameters for the Emission-Line Spectra of Extragalactic Objects, PASP, 93, 5 
Blandford, R. D., \& McKee, C. F. 1982, Reverberation Mapping of the Emission Line Regions of Seyfert Galaxies and Quasars, ApJ, 255, 419

Bellovary, J., Holley-Bockelmann, K., Gültekin, K., et al. 2014, Effects of Inclination on Measuring Velocity Dispersion and Implications for Black Holes, ApJ, in press (arXiv:1405.0286)

Bentz, M. C., Denney, K. D., Grier, C. J., Barth, A. J., Peterson, B. M., Vestergaard, M., Bennert, V. N., Canalizo, G., De Rosa, G., Filippenko, A. V., Gates, E. L., Greene, J. E., Li, W., Malkan, M. A., Pogge, R. W., Stern, D., Treu, T., \& Woo, J.-H. 2013, The Low-Luminosity End of the Radius-Luminosity Relationship for Active Galactic Nuclei, ApJ, 767, 149

Boroson, T. 2005, Blueshifted [O III] Emission: Indications of a Dynamic Narrow-Line Region, AJ, 130, 381

Cappellari, M., \& Emsellem, E. 2004, Parametric Recovery of Line-of-Sight Velocity Distributions from AbsorptionLine Spectra of Galaxies via Penalized Likelihood, PASP, 116,138

Choi, Y.-Y., Han, D.-H., \& Kim, S. S. 2010, Korea Institute for Advanced Study Value-Added Galaxy Catalog, JKAS, 43, 191

Crenshaw, D. M., Schmitt, H. R., Kraemer, S. B., Mushotzky, R. F., \& Dunn, J. P. 2010, Radial Velocity Offsets Due to Mass Outflows and Extinction in Active Galactic Nuclei, ApJ, 708, 419

Glikman, E., Helfand, D. J., White, R. L., et al. 2007, The FIRST-2MASS Red Quasar Survey, ApJ, 667, 673

Glikman, E., Urrutia, T., Lacy, M., et al. 2013, Dust Reddened Quasars in FIRST and UKIDSS: Beyond the Tip of the Iceberg, ApJ, 778, 127

Greene, J. E., \& Ho, L. C. 2004, Active Galactic Nuclei with Candidate Intermediate-Mass Black Holes, ApJ, 610, 722

Greene, J. E., \& Ho, L. C. 2005, Estimating Black Hole Masses in Active Galaxies Using the $H \alpha$ Emission Line, ApJ, 630, 122

Greene, J. E., \& Ho, L. C. 2007, A New Sample of Low-Mass Black Holes in Active Galaxies, ApJ, 670, 92

Haring, N., \& Rix, H. W. 2004, On the Black Hole MassBulge Mass Relation, ApJ, 604, L89

Heckman, T., \& Best, P. 2014, The Coevolution of Galaxies and Supermassive Black Holes: Insights from Surveys of the Contemporary Universe, ARAA, 52, 589

Hornschemeier, A. E., Heckman, T. M., Ptak, A. F., Tremonti, C. A., \& Colbert, E. J. M. 2005, ChandraSDSS Normal and Star-Forming Galaxies. I. X-Ray Source Properties of Galaxies Detected by the Chandra X-Ray Observatory in SDSS DR2, AJ, 129, 86

Kang, W.-R., Woo, J.-H., Schulze, A., et al. 2013, Calibrating Stellar Velocity Dispersions Based on Spatially Resolved H-Band Spectra for Improving the $M_{B H}-\sigma_{*}$ Relation ApJ, 767, 26

Kaspi, S., Smith, P. S., Netzer, H., Maoz, D., Jannuzi, B. T., \& Giveon, U. 2000, Reverberation Measurements for 17 Quasars and the Size-Mass-Luminosity Relations in Active Galactic Nuclei, ApJ, 533, 631

Kelly, B. C., \& Shen, Y. 2013, The Demographics of Broadline Quasars in the Mass-Luminosity Plane. II. Black Hole Mass and Eddington Ratio Functions, ApJ, 764, 45

Kewley, L. J., Groves, B., Kauffmann, G., \& Heckman, T. 2006, The Host Galaxies and Classification of Active Galactic Nuclei, MNRAS, 372, 961

Komossa, S., Xu, D., Zhou, H., Storchi-Bergmann, T., \& Binette, L. 2008, On the Nature of Seyfert Galaxies with
High [O III] 5007 Blueshifs, ApJ, 680, 926

Koratkar, A. P., \& Gaskell, C. M. 1991, Radius-Luminosity and Mass-Luminosity Relationship for Active Galatic Nuclei, ApJ, 370, L61

Kormendy, J., \& Ho, L. C. 2013, Coevolution (Or Not) of Supermassive Black Holes and Host Galaxies, ARAA, 51, 511

Land, K., Slosar, A., Lintott, C., Andreescu, D., Bamford, S., Murray, P., Nichol, R., Raddick, M. J., Schawinski, K., Szalay, A., Thomas, D., \& Vandenberg, J. 2008, Galaxy Zoo: the Large-Scale Spin Statistics of Spiral Galaxies in the Sloan Digital Sky Survey, MNRAS, 388, 1686

Lintott, C. J., Schawinski, K., Slosar, A., Land, K., Bamford, S., Thomas, D., Raddick, M. J., Nichol, R. C., Szalay, A., Andreescu, D., Murray, P., \& Vandenberg, J. 2008, Galaxy Zoo: Morphologies Derived from Visual Inspection of Galaxies from the Sloan Digital Sky Survey, MNRAS, 389, 1179

Lintott, C. J., Schawinski, K., Bamford, S., Slosar, A., Land, K., Thomas, D., Edmondson, E., Masters, K., Nichol, R. C., Raddick, M. J., Szalay, A., Andreescu, D., Murray, P., \& Vandenberg, J. 2011, Galaxy Zoo 1: Data Release of Morphological Classifications for Nearly 900000 Galaxies, MNRAS, 410, 166

Markwardt, C. B. 2009, Non-Linear Least-Squares Fitting in IDL with MPFIT, ASPC, 411, 251

McConnell, N. J., \& Ma, C.-P. 2013, Revisiting the Scaling Relations of Black Hole Masses and Host Galaxy Properties, ApJ, 764, 14

Nelson, C. H., \& Whittle, M. 1995, Stellar and Gaseous Kinematics of Seyfert Galaxies. I. Spectroscopic Data, ApJS, 99, 67

Netzer, H. 2009, Accretion and Star Formation Rates in Low-Redshift Type II Active Galactic Nuclei, MNRAS, 399, 1907

Oh, K., Sarzi, M., Schawinski, K., \& Yi, S. K. 2011, Improved and Quality-Assessed Emission and Absorption Line Measurements in Sloan Digital Sky Survey Galaxies, ApJS, 195, 13

Onken, C. A., Ferrarese, L., Merritt, D., Peterson, B. M., Pogge, R. W., Vestergaard, M., \& Wandel, A. 2004, Supermassive Black Holes in Active Galactic Nuclei. II. Calibration of the Black Hole Mass-Velocity Dispersion Relationship for Active Galactic Nuclei, ApJ, 615, 645

Park, D., Woo, J.-H., Treu, T., Barth, A. J., Bentz, M. C., Bennert, V. N., Canalizo, G., Filippenko, A. V., Gates, E., Greene, J. E., Malkan, M. A., \& Walsh, J. 2012, The Lick AGN Monitoring Project: Recalibrating Singleepoch Virial Black Hole Mass Estimates, ApJ, 747, 30

Reines, A. E., Greene, J. E., \& Geha, M. 2013, Dwarf Galaxies with Optical Signatures of Active Massive Black Holes, ApJ, 775, 116

Richards, G. T., Lacy, M., Storrie-Lombardi, L. J., Hall, P. B., Gallagher, S. C., Hines, D. C., Fan, X., Papovich, C., Vanden Berk, D. E., Trammell, G. B., Schneider, D. P., Vestergaard, M., York, D. G., Jester, S., Anderson, S. F., Budavári, T., \& Szalay, A. S. 2006, Spectral Energy Distributions and Multiwavelength Selection of Type 1 Quasars, ApJS, 166, 470

Schawinski, K., Urry, C. M., Virani, S., Coppi, P., Bamford, S. P., Treister, E., Lintott, C. J., Sarzi, M., Keel, W. C., Kaviraj, S., Cardamone, C. N., Masters, K. L., Ross, N. P., Andreescu, D., Murray, P., Nichol, R. C., Raddick, M. J., Solsar, A., Szalay, A. S., Thomas, D., \& Vandenberg, J. 2010, Galaxy Zoo: The Fundamentally Different Co- 
Evolution of Supermassive Black Holes and Their Earlyand Late-Type Host Galaxies, ApJ, 711, 284

Shen, J., Vanden Berk, D. E., Schneider, D. P., \& Hall, P. B. 2008, The Black Hole-Bulge Relationship in Luminous Broad-Line Active Galactic Nuclei and Host Galaxies, ApJ, 135, 928

Stern, J., \& Laor, A. 2012, Type I AGN at Low z. I. Emission Properties, MNRAS, 423, 600

Seyfert, C. K. 1943, Nuclear Emission in Spiral Nebulae, ApJ, 97, 28

Verolme, E. K., Cappellari M., Copin, Y., van der Marel, R. P., Bacon, R., Bureau, M., Davies, R. L., Miller, B. M., \& de Zeeuw, P. T. 2002, A SAURON Study of M32: Measuring the Intrinsic Flattening and the Central Black Hole Mass, MNRAS, 355, 517

Woo, J.-H., Treu, T., Malkan, M. A., \& Blandford, R. D. 2006, Cosmic Evolution of Black Holes and Spheroids. I. The $M_{B H}-\sigma_{*}$ Relation at $\mathrm{z}=0.36$, ApJ, 645, 900

Woo, J.-H., Treu, T., Barth, A. J., Wright, S. A., Walsh, J. L., Bentz, M. C., Martini, P., Bennert, V. N., Canal- izo, G., Filippenko, A. V., Gates, E., Greene, J., Li, W., Malkan, M. A., Stern, D., \& Minezaki, T. 2010, The LICK AGN Monitoring Project: The $M_{B H}-\sigma_{*}$ Relation for Reverberation-Mapped Active Galaxies, ApJ, 716,269

Woo, J.-H., \& Urry, C. M. 2002, Active Galactic Nucleus Black Hole Masses and Bolometric Luminosities, ApJ, 579,530

Woo, J.-H., Schulze, A., Park, D., Kang, W.-R., Kim, S. C., \& Riechers, D. A. 2013, Do Quiescent and Active Galaxies Have Different $M_{B H}-\sigma_{*}$ Relations?, ApJ, 772, 49

Wyithe, J. S. B., \& Loeb, J. 2002, A Physical Model for the Luminosity Function of High-Redshift Quasars, ApJ, 581,886

Xiao, T., Barth, A. J., Greene, J. E., Ho, L. C., Bentz, M. C., Ludwig, R. R., \& Jiang, Y. 2011, Exploring the Low-Mass End of the $M_{B H}-\sigma_{*}$ Relation with Active Galaxies, ApJ, 739, 28 